\title{
The role of hemostatic markers as venous stenosis or occlusion predictors following first transvenous cardiac device implantation
}

\author{
Andrzej Cacko ${ }^{1}$, Eliza Kozyra-Pydyśs ${ }^{2}$, Monika Gawałko², \\ Grzegorz Opolski², Marcin Grabowski ${ }^{2}$
}

${ }^{1}$ Department of Medical Informatics and Telemedicine, Medical University of Warsaw, Poland

${ }^{2} 1^{\text {st }}$ Department of Cardiology, Medical University of Warsaw, Poland

\begin{abstract}
Background: Among patients with an implanted cardiac implantable electronic device (CIED), ipsilateral upper extremity vein stenosis or occlusion (VSO) is observed more frequently than in the general population. However, there are no data available concerning the relationship between hemostatic markers (and their dynamics) and the occurrence of VSO. The aim of this study was to assess the predictive value of beta-thromboglobulin, the von Willebrand factor (vWF), fibrynogen and D-dimer for VSO development among first time CIED recipients.

Methods: This is a single-center, prospective study of consecutive first time CIED recipients without upper extremity VSO in baseline ultrasound examination. Biochemical data were collected from all the patients before CIED implantation (first measuring), up to 7 days subsequent (second measuring) and 6 months after the operation (third measuring). Primary endpoint was defined as the presence of upper extremity VSO at the implantation site during the ultrasound examination 6 months after the operation. Results: The study included 71 patients (mean age $73.1 \pm 10.5$ years; 39 [55\%] male). The incidence of VSO within 6-months follow up was $21.1 \%$. Average concentrations of hemostatic markers increased significantly in all patients immediately after CIED implantation. Serial hemostatic marker concentrations were similar in patients who met or did not meet the primary endpoint, apart from $v W F$. The mean concentration was significantly elevated in the group of 15 patients who reached the primary endpoint $(p=0.032)$.
\end{abstract}

Conclusions: A significant increase in $v W F$ concentration at 6 months post implantation may be a marker for VSO occurrence. (Cardiol J 2021; 28, 5: 690-696)

Key words: cardiac implanted electronic devices, vein stenosis or occlusion, hemostatic markers

\section{Introduction}

Among patients implanted with a cardiac implantable electronic device (CIED), ipsilateral upper extremity vein stenosis or occlusion (VSO) is observed more frequently than in the general population and occurs in $14-64 \%$ of patients with
CIED [1-3]. Although VSO is usually asymptomatic it can lead to upper extremity edema, paresthesia or pain and limits CIED upgrade.

Currently, several mechanisms of VSO formation are suggested. The most frequently mentioned one is the thromboembolic mechanism $[4,5]$. The postulated thromboembolic mechanism of VSO

Address for correspondence: Marcin Grabowski, MD, PhD, $1^{\text {st }}$ Department of Cardiology, Medical University of Warsaw, ul. Banacha 1a, 02-097 Warszawa, Poland, tel: +48 660751 816, fax: +48 2259919 57,

e-mail: marcin.grabowski@wum.edu.pl

Received: 28.09.2019 Accepted: 7.02.2019 Early publication date: 14.03.2019

This article is available in open access under Creative Common Attribution-Non-Commercial-No Derivatives 4.0 International (CC BY-NC-ND 4.0) license, allowing to download articles and share them with others as long as they credit the authors and the publisher, but without permission to change them in any way or use them commercially. 
formation prompts the search for biochemical indicators of pro-thrombotic activity, which would correlate with the risk of VSO.

The concentration of D-dimers is a biochemical marker of the thromboembolic process. The precursor of D-dimers is fibrinogen: one of the coagulation system proteins. It seems that among patients after CIED implantation, the concentration of $\mathrm{D}$-dimers and fibrinogen should be higher in patients with VSO [6]. Platelet activation results in secretion of many clotting activators, including beta-thromboglobulin (beta-TG). The von Willebrand factor (vWF), a glycoprotein involved in the hemostasis process, prevents the degradation of factor VIII of the coagulation pathway, promoting the formation of connections between collagen fibers, glycoproteins of the intercellular matrix and endothelial cells and blood platelets.

However, there are no specific data available concerning relationship between concentrations of the aforementioned hemostatic markers (and their dynamic) and the occurrence of VSO after CIED implantation. The aim of this study was to assess the predictive value of beta-TG, vWF, fibrynogen and D-dimer concentrations for VSO occurrence among first-time CIED recipients.

\section{Methods}

\section{Study population}

A single-center, prospective study was performed of consecutive first-time CIED recipients hospitalized in the documented department.

Patients included were those with:

- qualification for first-time intravenous implantation of the CIED system;

- written, informed consent to participate in the study.

Patients excluded were those with:

- upper extremity, shoulder girdle or jugular vein stenosis confirmed by preoperative imaging;

- venous compression syndromes of the upper extremity (thoracic outlet syndrome, cervical rib, compressive soft tissue tumors);

- thrombophilia;

- previous intervention on venous system at the intended implantation site.

\section{Clinical assessment and follow-up}

Each patient underwent an ultrasound examination to assess the condition of the venous system of the upper extremity, shoulder girdle and jugular veins before the planned operation and 6 months after the operation. The assessment of jugular veins and veins of shoulder girdle were performed in the supine position, while radiopaque and axillary veins were additionally assessed in the sitting position. A duplex Doppler mode consisting of a real-time B-mode image with a color-flow Doppler overlay was used for assessing the morphology and venous flow. All ultrasound examinations were carried out by experienced echocardiographers (all certified with the second-degree accreditation in echocardiography of the Echocardiography Working Group of the Polish Cardiac Society) using the Philips EnVisor C (Philips Electronics NV, Netherlands). The tests were examined using a 5-13 Mega-Hertz array transducer in both longitudinal and transverse sections.

All clinical conditions analyzed in the study, like diabetes or prediabetes, chronic heart failure, arterial hypertension, atrial fibrillation or atrial flutter, cancer, previous stroke or transient ischemic attack, were assessed based on subject medical history and in accordance with current guidelines.

The procedure of CIED implantation was performed in a reference cardiology unit by an expected electrophysiologist. For each subject the first-choice procedure to gain vascular access was venesection of cephalic vein. If this was unsuccessful, a subclavian vein puncture under ultrasound imaging was performed. Patient characteristics due to the number of implanted leads and type of vascular access was presented in a previous paper [7].

The concentrations of beta-TG, vWF, fibrynogen and $\mathrm{D}$-dimer were measured before CIED implantation (first measuring), up to 7 days subsequent (second measuring) and 6 months after the operation (third measuring). Manual EIA kits were used to measure beta-TG and vWF (Shanghai Sunred Biological Technology Co, Shanghai, China). Roche Diagnostics laboratory kits were used in order to conduct D-dimer and fibrynogen tests using Cobas 6000 analyzer.

\section{Study endpoints}

Primary endpoint was defined as the presence of VSO in the vein system of the upper extremity, shoulder girdle or jugular vein at the implantation site during the ultrasound examination 6 months after the operation. For veins accessible to direct insonation, the criteria of noncompressibility, visualization of echogenic intravascular mass, and the absence of respiratory variation were used (subclavian vein). For veins inaccessible to direct inosonation, the criterion of monophasic flow at the stenosis site with no retrograde wave or no 
Table 1. The mean concentrations of biochemical markers measured at $1^{\text {st }}, 2^{\text {nd }}$ and $3^{\text {rd }}$ measuring point in the whole study group.

\begin{tabular}{lcc}
\hline Hemostatic marker & Mean \pm SD & Median (IQR) \\
\hline Fibrynogen $[\mathrm{mg} / \mathrm{dL}]$ & $351.5 \pm 81.8$ & $343(86-530)$ \\
$1^{\text {st }}$ measuring & $424.3 \pm 95.5$ & $408(178-627)$ \\
$2^{\text {nd }}$ measuring & $404.2 \pm 98$ & $391(212-619)$ \\
$3^{\text {rd }}$ measuring & & $458(170-3210)$ \\
D-dimer [mg/dL] & $723.5 \pm 664$ & $875(326-6586)$ \\
$1^{\text {st }}$ measuring & $1252.1 \pm 1068.3$ & $766(230-3890)$ \\
$2^{\text {nd }}$ measuring & $1021.4 \pm 778.5$ & $12.25(3.78-27.56)$ \\
$3^{\text {rd }}$ measuring & & $16.64(5.37-66.55)$ \\
von Willebrand factor $[\mu \mathrm{g} / \mathrm{L}]$ & $13.26 \pm 5.55$ & $17.38(0.32-56.86)$ \\
$1^{\text {st }}$ measuring & $18.35 \pm 9.29$ & $13.43(3.42-30.37)$ \\
$2^{\text {nd }}$ measuring & $19.56 \pm 11.11$ & $18.18(2.21-34.18)$ \\
$3^{\text {rd }}$ measuring & & $10.06(5.61-32.7)$ \\
Beta-thromboglobulin $[\mu \mathrm{g} / \mathrm{L}]$ & $14.24 \pm 5.77$ & $18.12 \pm 6.93$ \\
$1^{\text {st }}$ measuring & $17.86 \pm 6.5$ & \\
$2^{\text {nd }}$ measuring & & \\
$3^{\text {rd }}$ measuring & & \\
\hline
\end{tabular}

Continuous and ordinal variables are shown as median (interquartile range [IQR]) and as mean \pm standard deviation (SD).

color signal or flow in the vessel lumen was used (middle part of subclavian, brachiocephalic vein) to detect VSO $[8,9]$.

\section{Statistical analysis}

Statistical analysis was performed using Statistica v. 12. Quantitative variables are expressed as mean \pm standard deviation and median (interquartile range). Categorical variables are presented as an exact number and percentage of patients. Differences between two groups for continuous variables were tested by the Mann-Whitney U-test. The comparisons of categorical variables were analyzed using the $\chi^{2}$ independence test. Two-way tables were assessed with the $\chi^{2}$ test with double-sided Fisher exact test due to a limited number of patients. A p value $<0.05$ was defined as statistically significant. The dynamics of biochemical marker changes were assessed using the Friedman test. Post hoc analysis with the Wilcoxon signed ranks test was performed using the Bonferroni correction for multiple comparisons ( 1 vs. 2,2 vs. 3,1 vs. 3 measuring point), resulting in a significance level set at $\mathrm{p}<0.017$.

\section{Results}

The study population consisted of 71 patients (mean age $73.1 \pm 10.5$ years; 39 [55\%] male).
Detailed patient characteristics were summarized in Supplemental Content (Suppl. Table S1). Implanted device systems comprised: cardioverter-defibrillator $(n=26)$, single-chamber or dual-chamber pacemakers $(n=34)$ and cardiac resynchronization therapy $(\mathrm{n}=11)$. The incidence of VSO within 6-month follow up was 15 (21.1\%) patients.

The mean concentrations of biochemical markers and their dynamics assessed at the $1^{\text {st }}, 2^{\text {nd }}$ and $3^{\text {rd }}$ measuring points in the whole study group are presented in Table 1 and Figure 1. The average concentration of each biochemical marker increased significantly between the $1^{\text {st }}$ and the $2^{\text {nd }}$ measuring points.

The average values of biochemical markers at all measuring points were similar among patients who met or did not meet primary endpoint, except for $\mathrm{vWF}$ concentrations at the $3^{\text {rd }}$ measuring point. The average concentration of the vWF 6 months after the CIED implantation was significantly greater in the group of patients with VSO than in the other subjects $(p=0.03)$. It was due to an additional increase of $\mathrm{vWF}$ concentration between the $2^{\text {nd }}$ and $3^{\text {rd }}$ measuring point observed only among patients with VSO (Fig. 2). The mean concentrations of biochemical markers and their dynamics in subgroups with and without primary endpoint were presented in Table 2 and Figure 2. 


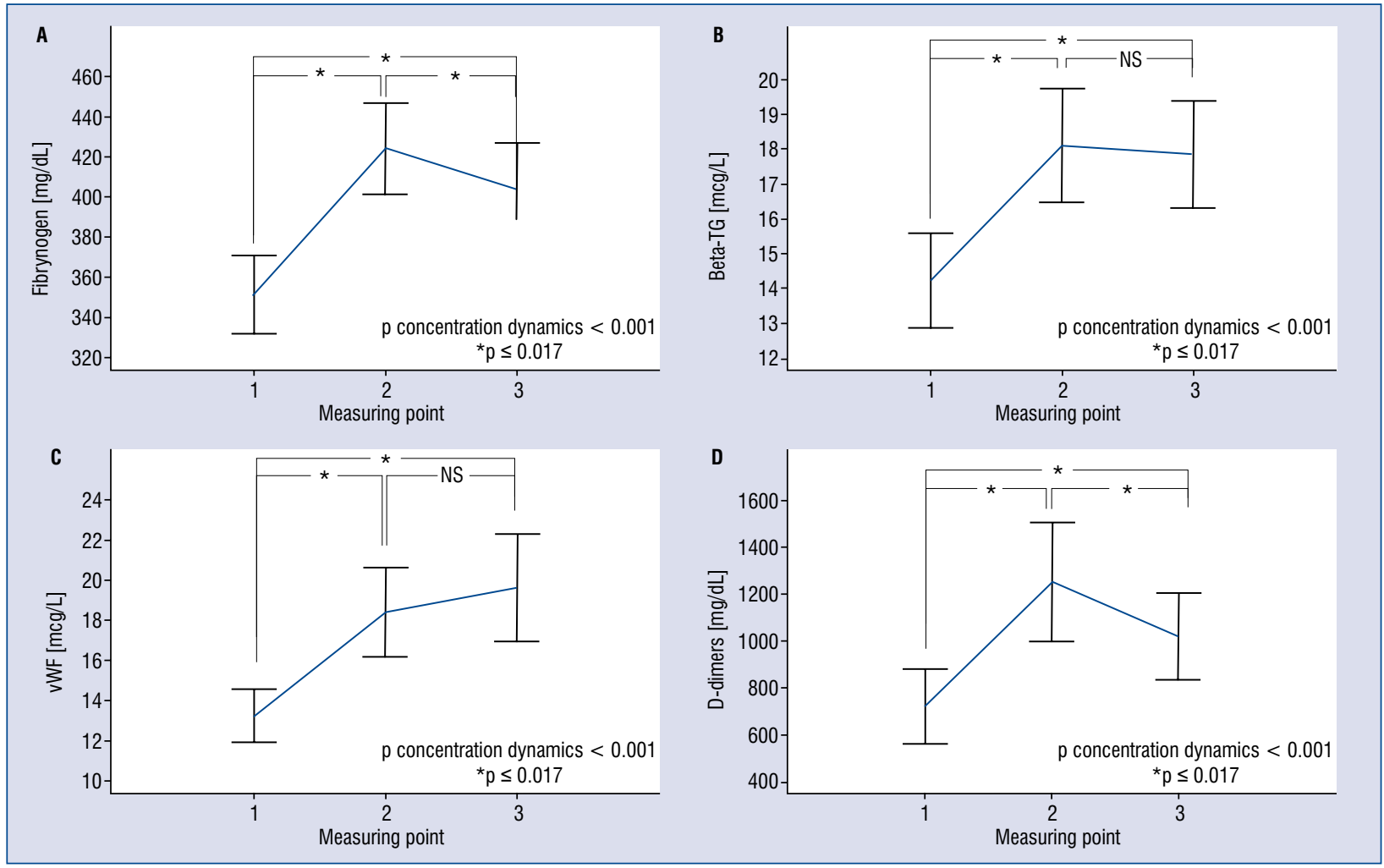

Figure 1. The mean concentrations of biochemical markers measured at $1^{\text {st }}, 2^{\text {nd }}$ and $3^{\text {rd }}$ measuring point in the whole study group; beta-TG — beta-thromboglobulin; vWF — the von Willebrand factor; NS — non-significant.

Table 2. The mean concentrations of biochemical markers measured at $1^{\text {st }}, 2^{\text {nd }}$ and $3^{\text {rd }}$ measuring point among patients who met or did not meet the primary endpoint.

\begin{tabular}{|c|c|c|c|c|c|}
\hline \multirow[t]{2}{*}{ Hemostatic marker } & \multicolumn{2}{|c|}{ Endpoint } & \multicolumn{2}{|c|}{ Non-endpoint } & \multirow[t]{2}{*}{$\mathbf{p}$} \\
\hline & Mean \pm SD & Median (IQR) & Mean \pm SD & Median (IQR) & \\
\hline \multicolumn{6}{|l|}{ Fibrynogen [mg/dL] } \\
\hline $1^{\text {st }}$ measuring & $347 \pm 87.5$ & $313(244-530)$ & $352.7 \pm 81$ & $352(86-481)$ & 0.61 \\
\hline $2^{\text {nd }}$ measuring & $410.7 \pm 91.3$ & $388(264-564)$ & $427.9 \pm 97$ & $413.5(178-627)$ & 0.54 \\
\hline $3^{\text {rd }}$ measuring & $398 \pm 100.6$ & $376(234-591)$ & $405.9 \pm 98.2$ & 396.5 (212-619) & 0.82 \\
\hline \multicolumn{6}{|l|}{ D-dimer [mg/dL] } \\
\hline $1^{\text {st }}$ measuring & $762.9 \pm 864.6$ & $424(170-3210)$ & $712.9 \pm 608.5$ & 458.5 (170-2908) & 0.58 \\
\hline $2^{\text {nd }}$ measuring & $1247.3 \pm 1132.6$ & 825 (357-4506) & $1253.4 \pm 1061.1$ & $929(326-6586)$ & 0.71 \\
\hline $3^{\text {rd }}$ measuring & $1091.1 \pm 949.7$ & $736(400-3890)$ & $1002.8 \pm 734.8$ & $766(230-3148)$ & 0.92 \\
\hline \multicolumn{6}{|c|}{ Von Willebrand factor $[\mu \mathrm{g} / \mathrm{L}]$} \\
\hline $1^{\text {st }}$ measuring & $14.62 \pm 5.77$ & $15.29(4.9-23.3)$ & $12.89 \pm 5.49$ & $11.7(3.78-27.56)$ & 0.31 \\
\hline $2^{\text {nd }}$ measuring & $16.54 \pm 6.85$ & $15.7(8.63-30.6$ & $18.83 \pm 9.84$ & $16.92(5.37-66.56)$ & 0.41 \\
\hline $3^{\text {rd }}$ measuring & $23.71 \pm 10.14$ & $18.79(14.67-42.5)$ & $18.45 \pm 11.19$ & $16.47(0.32-56.86)$ & 0.32 \\
\hline \multicolumn{6}{|c|}{ Beta-thromboglobulin $[\mu \mathrm{g} / \mathrm{L}]$} \\
\hline $1^{\text {st }}$ measuring & $16.2 \pm 8.58$ & $13.42(3.42-30.37)$ & $13.72 \pm 4.72$ & $13.52(4.09-25.75)$ & 0.64 \\
\hline $2^{\text {nd }}$ measuring & $18.31 \pm 8$ & $19.01(2.21-34.18)$ & $18.07 \pm 6.7$ & $17.59(3.03-34.14)$ & 0.77 \\
\hline $3^{\text {rd }}$ measuring & $18.11 \pm 6.63$ & $17.07(7.95-30.63)$ & $17.79 \pm 6.53$ & $17.04(5.61-32.7)$ & 0.91 \\
\hline
\end{tabular}

Continuous variables are shown as median (interquartile range [IQR]) and as mean \pm standard deviation (SD). P values are given for differences between the patients with and without primary endpoint. 


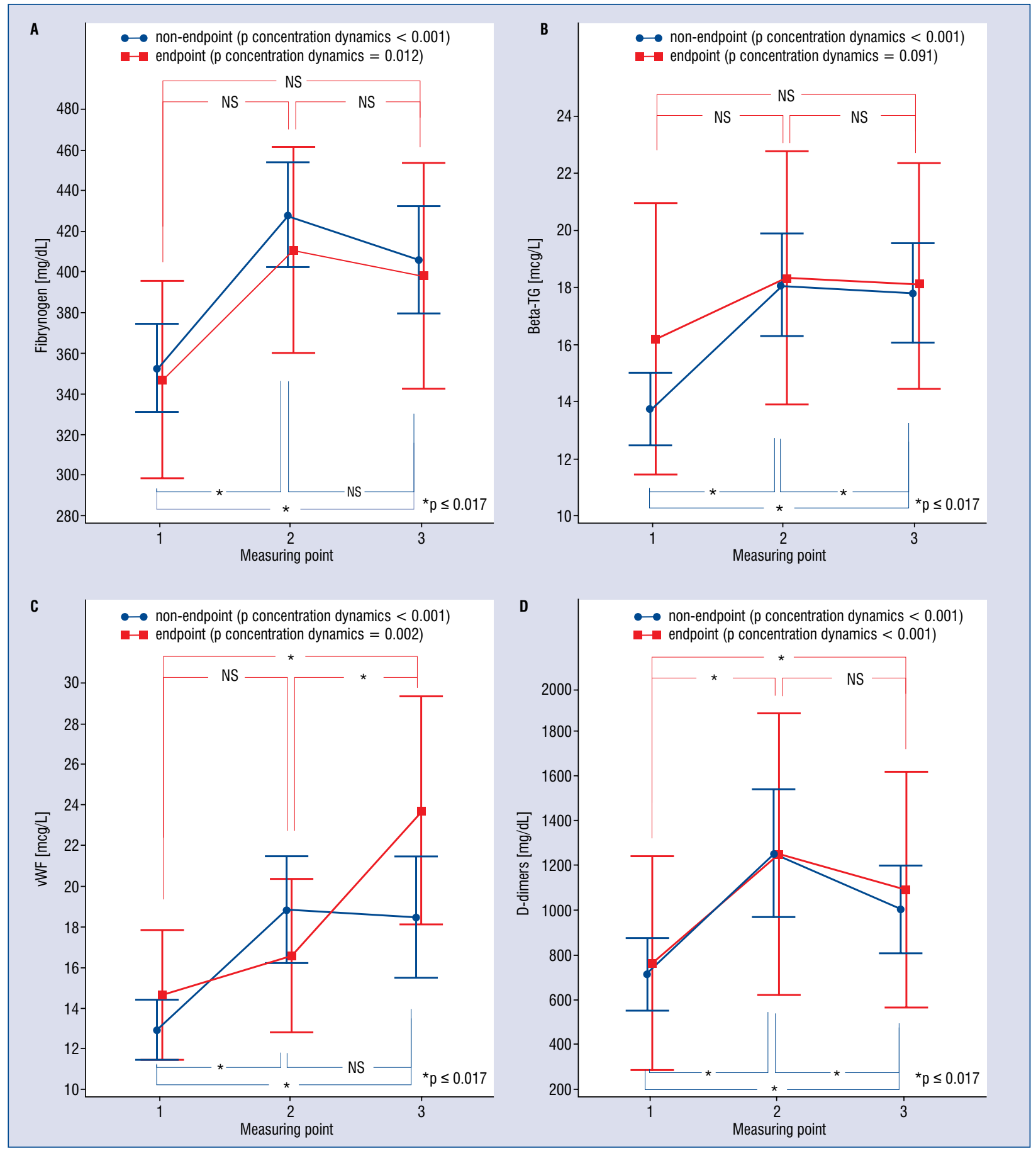

Figure 2. The mean concentrations of biochemical markers measured at the $1^{\text {st }}, 2^{\text {nd }}$ and $3^{\text {rd }}$ measuring point among patients who met or did not meet the primary endpoint; beta-TG — beta-thromboglobulin; vWF — the von Willebrand factor; NS - non-significant.

The observation that anticoagulation therapy or presence of cancer was not associated with an increased risk for primary endpoint occurrence as described in a previous publication [7].

\section{Discussion}

The present paper was focused on simultaneous analysis of the dynamics of concentrations 
of biochemical markers of inflammation (fibrinogen), coagulation activity (D-dimers) and platelet activation (vWF and beta-TG), in the population of first-time CIED recipients. According to available research, this is the first report describing dynamics of hemostatic markers among first-time CIED recipients followed-up by up to 6 months and their relationship with VSO, one of the most common complications of the lead placement into the vascular system. It should be emphasized that this report is important with regard to ensuring a better understanding of the mechanism of VSO occurrence and its prediction after CIED implantation.

Nevertheless, in starting the discussion, readers may be kindly forwarded to a previous paper presenting results of comparisons between subjects with and without study endpoints [6]. As it is a reallife population of first time CIED recipients, patients were included who had clinical conditions like atrial fibrillation, arterial hypertension, chronic heart failure, previous stroke or cancer. Though, during the follow up neither antithrombotic or anticoagulant treatment nor any of these conditions significantly increased the prevalence of VSO. Moreover, the presence of diabetes or prediabetes reduced the risk of VSO supporting the thesis of the inductive influence of inflammation. Reported observations built a multivariable prognostic model for VSO occurrence in the previously mentioned paper [7].

The procedure of CIED implantation, with intervention in the vascular system, initiated a significant increase in the concentration of biochemical markers. This is understandable considering the intervention itself (incision or puncture of a large venous vessel and preparation of the device pocket). However, only the vWF concentration, measured six months after the CIED implantation was significantly increased among patients who reached the primary endpoint. Moreover, the only marker that its concentration increased between the $2^{\text {nd }}$ and $3^{\text {rd }}$ measuring point among patients with VSO was vWF. It is worth mentioning that VSO occurrence is mostly associated with vessel trauma and subsequent inflammation [10]. This is consistent with the literature as vWF is synthesized in endothelium and is realized due to cell injury [11]. Moreover, inflammatory leukocytes release oxidizing agents that can render vWF more stable, with enhanced platelet binding, explaining higher concentrations of $\mathrm{vWF}$ among patients with VSO [12].

Results of this study propose possible clinical implantation of serial vWF measurement in a screening for VSO among first-time CIED recipients. Significant increases of vWF concentration between $7^{\text {th }}$ day and $6^{\text {th }}$ month follow-up from CIED implantation may identify patients with VSO. Still, as this is a pilot prospective study, additional observations in this field are required.

The fibrinogen and D-dimer concentrations have significantly decreased between $2^{\text {nd }}$ and $3^{\text {rd }}$ measuring points regardless of the occurrence of VSO. Also, the beta-TG concentration was reduced within 6 months (but not significantly). It is an important finding considering a conviction that promoted hemostasis and thrombosis should result in increase of fibrin-degradation-product concentration.

Finally, it is worth exploring the role of betaTG. This protein is stored in alpha-granules of platelets and is released in large amounts after platelet activation. It acts as a megakaryocyte maturation factor and helps in regulating platelet production, thus it has been recognized as a marker for activated platelets. Current studies suggest that an increased level of activated platelets, measured by higher plasma levels of beta-TG, is associated with increased risk of incidence of cardiovascular disease [13, 14]. For instance, the Plicner et al. [15] study included 108 consecutive patients undergoing coronary artery bypass grafting, demonstrated that increased platelet activation contributes to the occurrence of perioperative myocardial infarction in an early postoperative period. However, Kubota et al. [16], a study with 746 participants, do not support the hypothesis that higher concentrations of beta-TG reflect an increased risk of cardiovascular endpoints in the general population.

\section{Limitations of the study}

The present study is single-centered and nonrandomized. The size of the study population was the result of the test methodology (the study group encompassed only a population of first time CIED recipients) and the cost of biochemical markers and limited funding. Moreover, the study population is homogeneous as all of patients who underwent their first cardiac device implantation and were assessed exactly at 6 months postoperatively. Another limitation of this study is the single image approach to diagnose VSO. However, color Doppler ultrasonography is a non-invasive method with high sensitivity (80\%) and a specificity (90-100\%) for detecting VSO $[17,18]$. Another limitation is the fact that no attempt was made to study the ratio between the caliber of the vein and number of leads inserted. 


\section{Conclusions}

All biochemical hemostatic marker levels increased significantly in response to transvenous CIED insertion and the presence of electrodes in the venous system. A significant increase in vWF level at 6 months post implantation may be a marker of VSO occurrence.

Funding: This work was supported by a scientific grant of the Polish Cardiac Society.

\section{Conflict of interest: None declared}

\section{References}

1. Raatikainen MJ, Arnar DO, Zeppenfeld K, et al. Statistics on the use of cardiac electronic devices and electrophysiological procedures in the European Society of Cardiology countries: 2014 report from the European Heart Rhythm Association. Europace. 2015; 17 Suppl 1: i1-75, doi:10.1093/europace/euu300, indexed in Pubmed: 25616426.

2. Santini M, Di Fusco SA, Santini A, et al. Prevalence and predictor factors of severe venous obstruction after cardiovascular electronic device implantation. Europace. 2016; 18(8): 1220-1226, doi: 10.1093/europace/euv391, indexed in Pubmed: 26705557.

3. Jonik S, Grabowski M, Pietura R, et al. Successful removal of stented leads by using percutaneous approach. Heart Beat J. 2017; 2: 22-26, doi: 10.24255/hbj/81161.

4. Rozmus G, Daubert JP, Huang DT, et al. Venous thrombosis and stenosis after implantation of pacemakers and defibrillators. J Interv Card Electrophysiol. 2005; 13(1): 9-19, doi: 10.1007/ s10840-005-1140-1, indexed in Pubmed: 15976973.

5. Lickfett L, Bitzen A, Arepally A, et al. Incidence of venous obstruction following insertion of an implantable cardioverter defibrillator. A study of systematic contrast venography on patients presenting for their first elective ICD generator replacement. Europace. 2004; 6(1): 25-31, indexed in Pubmed: 14697723.

6. Pacholewicz J, Kuliczkowski W, Kaczmarski J, et al. Activated hemostatic biomarkers in patients with implanted left ventricle assist devices: are heparin and/or clopidogrel justified? Cardiology. 2015 ; 131(3): 172-176, doi: 10.1159/000375232, indexed in Pubmed: 25967953.

7. Cacko A, Kozyra-Pydyś E, Gawałko M, et al. Predictors of venous stenosis or occlusion following first transvenous cardiac device implantation: Prospective observational study. J Vasc Access. 2018 [Epub ahead of print]: 1129729818815135, doi: 10.1177/1129729818815135, indexed in Pubmed: 30537896.
8. van Rooden CJ, Rosendaal FR, Barge RMY, et al. Central venous catheter related thrombosis in haematology patients and prediction of risk by screening with Doppler-ultrasound. $\mathrm{Br}$ J Haematol. 2003; 123(3): 507-512, indexed in Pubmed: 14617015.

9. Debourdeau P, Espié M, Chevret S, et al. Incidence, risk factors, and outcomes of central venous catheter-related thromboembolism in breast cancer patients: the CAVECCAS study. Cancer Med. 2017; 6(11): 2732-2744, doi: 10.1002/cam4.1201, indexed in Pubmed: 28980454.

10. Agarwal AK. Central vein stenosis: current concepts. Adv Chronic Kidney Dis. 2009; 16(5): 360-370, doi: 10.1053/j. ackd.2009.06.003, indexed in Pubmed: 19695504.

11. Chauhan AK, Kisucka J, Lamb CB, et al. von Willebrand factor and factor VIII are independently required to form stable occlusive thrombi in injured veins. Blood. 2007; 109(6): 24242429, doi: 10.1182/blood-2006-06-028241, indexed in Pubmed: 17119108.

12. Fu X, Chen J, Gallagher R, et al. Shear stress-induced unfolding of VWF accelerates oxidation of key methionine residues in the A1A2A3 region. Blood. 2011; 118(19): 5283-5291, doi: 10.1182/ blood-2011-01-331074, indexed in Pubmed: 21917758.

13. Sharma G, Berger JS. Platelet activity and cardiovascular risk in apparently healthy individuals: a review of the data. J Thromb Thrombolysis. 2011; 32(2): 201-208, doi: 10.1007/s11239-0110590-9, indexed in Pubmed: 21562837.

14. Migliorini A, Valenti R, Marcucci R, et al. High residual platelet reactivity after clopidogrel loading and long-term clinical outcome after drug-eluting stenting for unprotected left main coronary disease. Circulation. 2009; 120(22): 2214-2221, doi: 10.1161/CIRCULATIONAHA.109.883454, indexed in Pubmed: 19917884.

15. Plicner D, Ziętkiewicz M, Mazur P, et al. Beta-thromboglobulin as a marker of perioperative myocardial infarction in patients undergoing coronary artery bypass grafting following aspirin discontinuation. Platelets. 2014; 25(8): 603-607, doi: 10.3109/09 537104.2013.854877, indexed in Pubmed: 24433129.

16. Kubota Y, Alonso A, Heckbert SR, et al. Beta-thromboglobulin and incident cardiovascular disease risk: The Atherosclerosis Risk in Communities study. Thromb Res. 2017; 155: 116-120, doi: 10.1016/j.thromres.2017.05.016, indexed in Pubmed: 28531882.

17. van Rooden CJ, Molhoek SG, Rosendaal FR, et al. Incidence and risk factors of early venous thrombosis associated with permanent pacemaker leads. J Cardiovasc Electrophysiol. 2004; 15(11): 1258-1262, doi: 10.1046/j.1540-8167.2004.04081.x, indexed in Pubmed: 15574174.

18. Zuber M, Huber P, Fricker U, et al. Assessment of the subclavian vein in patients with transvenous pacemaker leads. Pacing Clin Electrophysiol. 1998; 21(12): 2621-2630, indexed in Pubmed: 9894653. 